\title{
Novo método de cálculo das frações de verde para controle semafórico em tempo real
}

\author{
Werner Kraus Junior ${ }^{1}$, José Vergara Dietrich ${ }^{2}$, \\ Felipe Augusto de Souza ${ }^{3}$ e Eduardo Camponogara ${ }^{4}$
}

\begin{abstract}
Resumo: Apresenta-se um método de cálculo de frações de verde a ser usado em sistemas de controle de tráfego em tempo real por amostragem cíclica. A base do método origina-se da estratégia TUC, brevemente revisada neste trabalho. O objetivo é substituir um procedimento empírico de ajuste de parâmetros auxiliares do controle por uma metodologia que seja facilmente entendida e utilizável na prática. Resultados de simulação indicam o desempenho superior do método quando comparado com os ajustes empíricos do método original.

Palavras-chave: controle semafórico em tempo real; amostragem cíclica; método TUC.
\end{abstract}

\begin{abstract}
A new method for the computation of splits for real-time traffic control with cyclic sampling is presented. The method is based on the TUC strategy, which is briefly reviewed in this paper. The goal is to replace an empirical tuning procedure of auxiliary control parameters by a method that is easy to understand and implement. Simulation results indicate the superior performance of the method when compared to the empirical adjustments of the original method.
\end{abstract}

Keywords: real-time traffic control; cyclic sampling; TUC method.

\section{INTRODUÇÃO}

Cruzamentos semaforizados são uma solução simples e eficaz para melhorar a segurança no trânsito. Apresentam elevada eficiência em situações de volumes de tráfego altos quando comparados a cruzamentos com regras de prioridade. Além disso, podem ser aplicados sem mudanças drásticas de geometria e em espaços restritos, ao contrário de soluções de maior capacidade e de custo mais alto como túneis e viadutos. Devido a tais vantagens, os semáforos são largamente usados no controle do tráfego nas cidades.

Com os semáforos, surge o problema de ajuste dos tempos de abertura para cada movimento do tráfego que garanta a máxima eficiência possível na operação viária. Vários métodos existem para a solução do problema; Gordon e Tighe (2005) usam seis categorias para classificar as diversas soluções de acordo com área abrangida (desde cruzamentos isolados até redes urbanas) e dos métodos empregados. Entretanto, se apenas a forma de cálculo dos tempos for considerada, quatro categorias podem ser definidas: (i) tempo fixo, no qual os tempos de abertura são pré-determinados e fixos, mudando de acordo com a hora do dia ou com o padrão vigente de tráfego; (ii) atuados, que alteram os tempos fixos com base em eventos detectados na vizinhança do cruzamento (p. ex., pedestre acionando a botoeira para travessia); (iii) reativos, nos quais os tempos são calculados

\footnotetext{
${ }^{1}$ Werner Kraus Junior, Departamento de Automação e Sistemas, Universidade Federal de Santa Catarina, Florianópolis, SC, Brasil. (e-mail: werner@das.ufsc.br).

2 José Dolores Vergara Dietrich, Coordenação de Engenharia Eletrônica, Universidade Tecnológica Federal do Paraná, Campus Toledo, Toledo, PR Brasil. (e-mail: vergara@utfpr.edu.br).

${ }^{3}$ Felipe Augusto de Souza, Programa de Pós-Graduação em Automação e Sistemas, Universidade Federal de Santa Catarina, Florianópolis, SC, Brasil. (e-mail: fas@das.ufsc.br).

${ }^{4}$ Eduardo Camponogara, Departamento de Automação e Sistemas, Universidade Federal de Santa Catarina, Florianópolis, SC, Brasil. (e-mail camponog@das.ufsc.br).
}

Manuscrito recebido em 21/7/2010 e aprovado para publicação em 3/3/2011 Este artigo é parte de TRANSPORTES v.19, n.1, 2011. ISSN: 2237-1346 (online). online a partir de dados de sensores, ou seja, usando informações atualizadas do estado do tráfego; e (iv) adaptativos, que usam técnicas preditivas do estado futuro do tráfego no cálculo dos tempos. As duas últimas categorias também são chamadas de métodos em "tempo real", pois os tempos de verde são calculados somente no momento em que serão aplicados nos semáforos.

Diversos estudos apontam as vantagens dos sistemas em tempo real, como, por exemplo, a recente síntese de diversas experiências na implantação e operação de tais sistemas encontrada em NCHRP (2010). Ganhos de desempenho operacional são maiores quando comparados com planos de tempos fixos ajustados em campo, pois frequentemente tais ajustes são sub-ótimos (Kraus et al., 2010).

Neste trabalho, são propostas melhorias no sistema reativo de tempo real Traffic-responsive Urban Control TUC (Diakaki et al., 2002, 2003), o qual tem apresentado desempenho similar a sistemas consagrados tais como SCOOT (Robertson e Bretherton, 1991) em testes de campo (Kosmatopoulos et al., 2006). O foco é o controle de frações de verde (ou splits, em inglês). Busca-se substituir o ajuste ad hoc de parâmetros de controle por uma metodologia baseada em princípios de engenharia de tráfego. Nota-se que os ajustes de parâmetros são necessários em sistemas de tempo real, sejam reativos ou adaptativos, contribuindo para custos adicionais e necessidade de manutenção ao longo do tempo (NCHRP, 2010). Portanto, ao formular um método que dispensa tais ajustes, este trabalho contribui para o barateamento e facilitação da implantação e uso de sistemas em tempo real.

O novo método de cálculo das frações de verde apresenta, nos casos estudados, desempenho superior ao método TUC. Ainda assim, os mecanismos básicos do TUC são preservados, com especial destaque à técnica de amostragem cíclica do tráfego e do projeto de controle baseado na teoria de reguladores lineares quadráticos, ou LQR (sigla para o inglês Linear Quadratic Regulator) (Dorato et al., 1995). 


\section{AMOSTRAGEM CÍCLICA}

Este trabalho baseia-se na filosofia de controle de tráfego apresentada pela estratégia TUC, da qual retém dois elementos essenciais: a amostragem cíclica do estado do tráfego e o projeto inicial via metodologia LQR. Esta seção explora em mais detalhes ambas as ideias.

\subsection{Vantagens da amostragem cíclica}

Do ponto de vista metodológico, a grande inovação trazida pelo método TUC é o uso de períodos de amostragem do tráfego iguais ao ciclo vigente. Isto é, apenas uma amostra a cada ciclo é obtida dos detectores instalados nas vias. A única variável amostrada é a ocupação temporal dos detectores, isto é, quanto tempo durante um ciclo cada detector ficou ocupado por veículos. A partir da informação da ocupação, estima-se o número de veículos por ciclo com base em funções não lineares calibradas de acordo com a distância dos detectores até a linha de parada (Diakaki, 1999).

Tal abordagem distingue-se das demais estratégias de tempo real que operam a partir da construção de modelos de filas baseados na amostragem do tráfego em períodos curtos de tempo (da ordem de unidades de segundos). As principais vantagens do método de amostragem cíclica, tanto do ponto de vista teórico como prático, são:

- O conceito de amostragem cíclica do tráfego simplifica a estimação do estado das vias, pois elimina a necessidade de definir o que vem a ser uma "fila" e, por conseguinte, os estados de tais filas. Intuitivamente, parece evidente caracterizar uma fila de tráfego; porém, vários estudos (Gallego et al., 1997; Baras et al., 1979) apontam para a precariedade do conceito, uma vez que veículos trafegando em velocidades muito abaixo da velocidade de fluxo livre da via poderiam, mesmo não estando parados, ser considerados como aguardando a abertura do sinal e, portanto, em "fila". Assim, em lugar de tentar estimar as "filas" ou, equivalentemente, os perfis cíclicos do tráfego como no caso do sistema SCOOT, usa-se apenas o número estimado de veículos em um ciclo semafórico, não importando o estado em que se encontram;

- O tratamento da informação de detectores para fins de implementação torna-se mais robusto, pois não depende da calibração fina de modelos de tráfego como em métodos consagrados de amostragem de alta frequência, isto é, com período da ordem de segundos ou décimos de segundo. Exemplos de tais abordagens são SCOOT (Robertson e Bretherton, op. cit.), RHODES (Mirchandani e Head, 2001) e OPAC (Gartner, 1985);

- O cálculo das frações de verde por amostragem cíclica apresenta tolerância intrínseca a atrasos variáveis na comunicação entre controladores e a central de controle, pois faz pouca diferença relativa receber um dado com atraso de alguns segundos quando se trata de ciclos semafóricos com duração da ordem de minuto;

- Por último, conforme já discutido anteriormente, o desempenho resultante em instalações de campo da estratégia TUC que usa amostragem cíclica é similar a de outros sistemas consagrados de controle em tempo real e, portanto, superior ao tempo fixo, pois esse não é capaz de ajustar-se de maneira precisa às condições vigentes do tráfego.

A seguir, apresenta-se resumidamente o cálculo de frações de verde pelo método TUC. A extensa teoria subjacen- te está além do escopo deste texto. Assim, é dada ênfase na interpretação da matriz de controle usada no cálculo.

\subsection{Cálculo das frações de verde no método TUC}

Tendo sido originalmente projetado para operar em condições momentaneamente sobre-saturadas de tráfego, o principal objetivo do controle de frações de verde na estratégia TUC é a redução do risco de transbordamento da capacidade de estocagem de veículos no link e, por conseguinte, evitar o bloqueio de cruzamentos. Em condições de tráfego saturado, é razoável supor que os tempos de verde são inteiramente usados pelos veículos. Portanto, passam a valer as condições de modelos "estocagem-e-progressão" (storeand-forward, em inglês), que consideram que é possível descrever a evolução dos volumes de tráfego em cada segmento das vias apenas com base na informação de tempos de abertura de verdes (uma vez que estes são totalmente utilizados) (Diakaki et al., 2002). Ou seja, nessas condições a dinâmica do tráfego pode ser descrita como uma equação vetorial linear, invariante no tempo e discreta com a forma:

$$
\mathrm{x}(k+1)=\mathrm{x}(k)+\mathrm{B} \Delta \mathrm{g}(k)
$$

em que,

$k$ : índice da amostra temporal (tomada a cada ciclo);

$\mathrm{x}$ : vetor com o número de veículos $x_{z}$ no segmento $z \in Z$ (conjunto de todos os segmentos sendo controlados);

$\Delta \mathrm{g}: \quad$ vetor contendo os tempos de verde $\Delta g_{j, i}=g_{j, i}-$ $g_{j, i}^{N}, \forall i \in F_{j}$ (conjunto das fases de um semáforo), $\forall j \in J$ (conjunto dos cruzamentos controlados); e

$g_{j, i}$ : tempo de verde da fase $i$ no cruzamento $j$ e $g_{j, i}^{N}$ é um valor invariante de verde nominal definido pelo projetista.

A matriz B contém os parâmetros que refletem a topologia da rede, mais especificamente, os fluxos de saturação, a estrutura das fases (relacionando quais verdes acrescentam ou subtraem veículos de cada segmento), e as taxas de conversão.

A interpretação de (1) pode ser feita nos seguintes termos: uma vez que a matriz B é invariante, no próximo instante de tempo $(k+1)$ o número de veículos em cada segmento $\mathrm{x}(k+1)$ será igual ao número atual $(\mathrm{x}(k))$ acrescido ou subtraído de quantidades de veículos que só dependem da duração dos verdes $g_{j, i}$. No caso de $g_{j, i}=g_{j, i}^{N}-\Delta g_{j, i}$ torna-se zero e o sistema de tráfego permanece em equilíbrio $(\mathrm{x}(k+1)=\mathrm{x}(k))$.

Evidentemente, a dinâmica do tráfego descrita por (1) é muito simplificada, falhando nos casos em que os verdes não são totalmente aproveitados (caso sub-saturado) ou quando variam muito os fluxos de saturação devido ao congestionamento, pois nesse caso B teria de ser variante no tempo. De todo modo, a hipótese básica dos modelos storeand-forward supõe que os veículos possam passar de um segmento ao seguinte no intervalo $(k ; k+1)$ com taxa de descarga igual ao fluxo de saturação do modelo. Embora a hipótese pareça muito restritiva, o modelo resultante consegue capturar a essência dos parâmetros da malha viária necessários para o projeto de controle realimentado. 
Vale ressaltar também a hipótese de que a matriz B é invariante. As principais características do tráfego que se refletem nos elementos de B são as taxas de conversão e os fluxos de saturação. Ora, ambas são, na verdade, variantes no tempo. Portanto, na prática adotam-se valores médios para estas características. As medições cíclicas do tráfego e o cálculo realimentado do controle são usados para compensar as variações paramétricas do sistema de tráfego. Entretanto, caso tais variações sejam extremas a ponto de prejudicar o desempenho do controle, pode-se usar vários modelos para diferentes horários do dia.

Baseado no modelo linear (1), a metodologia de projeto LQR (Dorato et al., op. cit.) é então empregada para síntese de uma lei de controle da forma:

$$
\mathrm{g}(k)=\mathrm{g}^{N}-\mathrm{Lx}(k)
$$

em que,

$$
\begin{aligned}
\mathrm{g}: & \text { vetor dos tempos de verde } g_{j, i} ; \\
\mathrm{g}^{N}: & \text { vetor dos tempos de verde nominais escolhidos } \\
& \text { pelo projetista; e } \\
\mathrm{L}: & \text { matriz de ganhos que resulta do projeto via LQR. }
\end{aligned}
$$

Embora fuja do escopo deste trabalho, acrescenta-se que a metodologia LQR permite ao projetista levar em conta a capacidade de armazenamento dos segmentos (caixas) das vias.

A aplicação do controle (2) em tempo real exige o conhecimento do número de veículos $x_{z}(k)$ no ciclo atual para cômputo dos tempos de verde. Para tanto, as medidas de ocupações são usadas na estimação do número de veículos através do uso de funções não-lineares $x_{z}=f_{z}\left(o_{z}(k)\right)$. Isto é, em vez de tentar estimar com precisão as filas de tráfego, o método TUC usa mapeamentos $f_{z}(\cdot)$ que fornecem estimativas do número de veículos acumulados em um ciclo a partir dos valores de ocupação. Calibra-se $f_{z}(\cdot)$ para refletir o posicionamento do detector no segmento; quanto mais próximo da linha de parada, menor o número de veículos que será estimado para uma dada ocupação. Como forma de estabelecer um compromisso entre a detecção prematura do crescimento de filas e a habilidade de medir fluxos (em vez de veículos parados), recomenda-se o posicionamento dos detectores no meio da quadra entre dois cruzamentos (Diakaki, 1999).

Para exemplificar a aplicação do método LQR na síntese do controle, considere a malha viária mostrada na Figura 1, a qual será usada também em simulações. Os rótulos dos cruzamentos e segmentos de via serão usados no restante

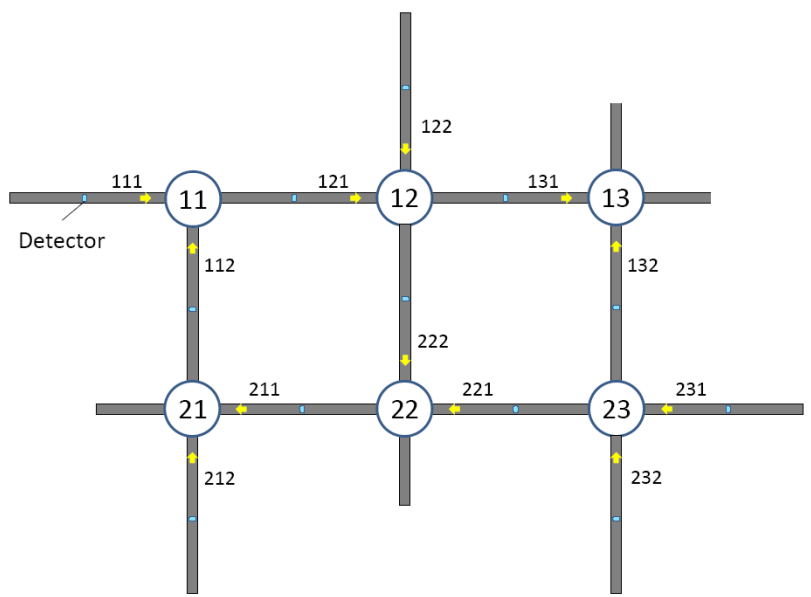

Figura 1. Malha viária I, com vias de uma faixa e de mão única

do texto. Têm-se seis cruzamentos de vias de mão única e faixa simples com as seguintes características:

- Comprimento dos segmentos: $150 \mathrm{~m}$;

- Fluxo de saturação: $1800 \mathrm{vph}$;

- Taxas de conversão: $100 \%$ dos veículos seguem em frente; e

- Detectores: $75 \mathrm{~m}$ da linha de parada.

Com tais características, a lei de controle projetada via LQR tem a forma apresentada na Equação (3). Esta equação revela que o controle, neste caso particular, apresenta apenas $\{0,-2\}$ como elementos de $L$. Assim, é fácil ver a alocação de $2 \mathrm{~s}$ por veículo no cômputo das frações de verde, pois os elementos de $L$ multiplicam as quantidades de veículos $x_{i j l}$. A forma esparsa da matriz reflete a simplicidade da malha viária e a ausência de giros do tráfego; as linhas de $L$ indicam claramente quais estimativas de $x_{z}$ vão afetar as frações de verde $g_{i j l}$.

\section{CARACTERIZAÇÃO DO PROBLEMA DE CONTROLE}

Na seção anterior foi apresentada a forma básica de controle pelo método LQR. Em aplicações práticas, é preciso realizar ajustes em parâmetros auxiliares para obter-se um bom desempenho do controle, conforme discutido a seguir.

$$
\left[\begin{array}{l}
g_{111} \\
g_{112} \\
g_{121} \\
g_{122} \\
g_{131} \\
g_{132} \\
g_{211} \\
g_{212} \\
g_{221} \\
g_{222} \\
g_{231} \\
g_{232}
\end{array}\right]=\left[\begin{array}{cccccccccccc}
-2 & 0 & 0 & 0 & 0 & 0 & 0 & 0 & 0 & 0 & 0 & 0 \\
0 & -2 & 0 & 0 & 0 & 0 & 0 & -2 & 0 & 0 & 0 & 0 \\
-2 & 0 & -2 & 0 & 0 & 0 & 0 & 0 & 0 & 0 & 0 & 0 \\
0 & 0 & 0 & -2 & 0 & 0 & 0 & 0 & 0 & 0 & 0 & 0 \\
-2 & 0 & -2 & 0 & -2 & 0 & 0 & 0 & 0 & 0 & 0 & 0 \\
0 & 0 & 0 & 0 & 0 & -2 & 0 & 0 & 0 & 0 & 0 & -2 \\
0 & 0 & 0 & 0 & 0 & 0 & -2 & 0 & -2 & 0 & -2 & 0 \\
0 & 0 & 0 & 0 & 0 & 0 & 0 & -2 & 0 & 0 & 0 & 0 \\
0 & 0 & 0 & 0 & 0 & 0 & 0 & 0 & -2 & 0 & -2 & 0 \\
0 & 0 & 0 & -2 & 0 & 0 & 0 & 0 & 0 & -2 & 0 & 0 \\
0 & 0 & 0 & 0 & 0 & 0 & 0 & 0 & 0 & 0 & -2 & 0 \\
0 & 0 & 0 & 0 & 0 & 0 & 0 & 0 & 0 & 0 & 0 & -2
\end{array}\right]\left[\begin{array}{l}
x_{111} \\
x_{112} \\
x_{121} \\
x_{122} \\
x_{131} \\
x_{132} \\
x_{211} \\
x_{212} \\
x_{221} \\
x_{222} \\
x_{231} \\
x_{232}
\end{array}\right]
$$




\subsection{Descrição da simulação e dos controles de Tempo Fixo e LQR}

Para demonstração do problema de controle que se busca resolver, emprega-se o ambiente de simulação microscópica Aimsun (TSS, 2009), cujo modelo de perseguição veicular é inspirado no trabalho de Gipps (Gipps, 1981). Por se tratar de estudo comparativo de estratégias de controle, não será feita a calibração de malhas viárias, optando-se pelo uso das malhas apresentada na Figura 1. Para fins de implementação do controle, são usados recursos de interfaceamento do Aimsun com módulos de controle externos escritos em linguagem Python disponíveis em plataforma projetada para facilitar o teste de estratégias de controle complexas (Souza et al., 2010).

O modelo da malha viária é construído de acordo com os fluxos de saturação, comprimentos de vias, posição de detectores e taxas de conversão apresentados na Seção 2.2. Os volumes de tráfego são de $750 \mathrm{vph}$ em todas as cinco aproximações de entrada da malha viária. O tempo de ciclo é fixo, de $70 \mathrm{~s}$, e não há defasagens entre os cruzamentos, uma vez que o objetivo é o estudo do efeito das frações de verde no desempenho do controle. Os tempos de amarelo são de $4 \mathrm{~s}$, e os vermelhos totais duram $2 \mathrm{~s}$, resultando em entreverdes de $6 \mathrm{~s}$. Duas estratégias são consideradas:

- LQR, conforme descrito pela Eq. (3); e

- Tempo Fixo, com frações idênticas para cada aproximação.

As taxas de chegadas são determinísticas com headways constantes. Tal padrão de chegadas serve para destacar o fenômeno que se deseja estudar. Cada simulação dura 2 h,

Cruzamento 21

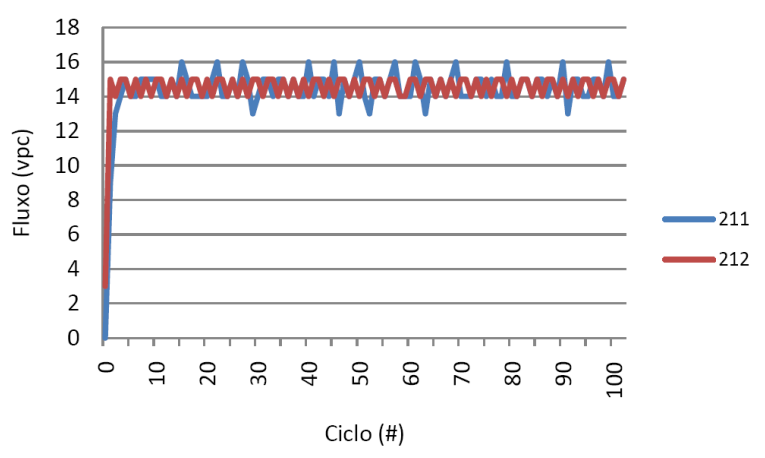

(a) Fluxos (veículos por ciclo) no cruzamento 21, tempo fixo

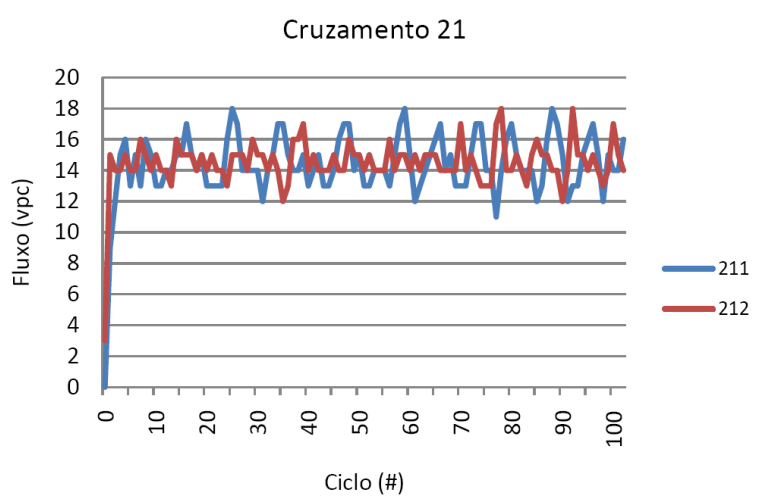

(c) Fluxos (veículos por ciclo) no cruzamento 21, LQR sem período de warm-up para que se evidencie o comportamento transitório do controle realimentado no início do carregamento da malha viária.

É importante destacar que, nas condições descritas acima, as frações de verde do controle Tempo Fixo adotado são ótimas no sentido de apresentar atraso mínimo para ciclo fixo de $70 \mathrm{~s}$. Isso porque as capacidades e os volumes de tráfego são idênticos em todas as aproximações dos cruzamentos. Portanto, as taxas de serviço (fluxo/fluxo de saturação) são sempre iguais, e o ajuste ótimo consiste em distribuir igualmente os tempos de verde (Webster, 1969). Ou seja, o melhor que o controle LQR deveria fazer é convergir para frações iguais e constantes.

\subsection{Comparação de desempenho para taxa de chegadas constantes}

Para ilustrar o comportamento do controle LQR, a Figura 2 apresenta os resultados de simulação para o cruzamento 21 , compostos pelos segmentos 211 e 212 . Claramente, o resultado com controle por tempo fixo é melhor do que o obtido com LQR. O mau desempenho está associado ao surgimento de oscilações no controle, conforme mostra o gráfico da Figura 2.

O surgimento das oscilações no controle LQR deve-se à forma com que os elementos da matriz $L$ ponderam os fluxos estimados de veículos no cálculo das frações de verde. Considerando o cruzamento em questão (21), a partir da forma de $L$ na Eq. (3), pode-se perceber que a fração de verde do segmento 212 está em desvantagem em relação à 211, pois:

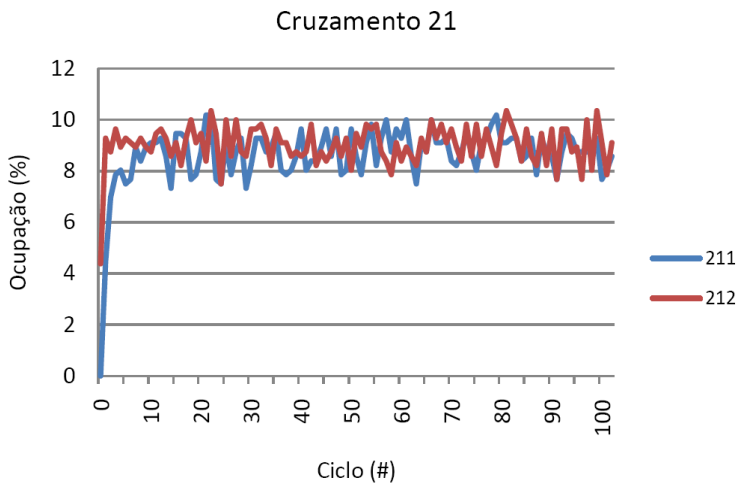

(b) Ocupações do detector em relação ao ciclo (\%), cruzamento 21 , tempo fixo

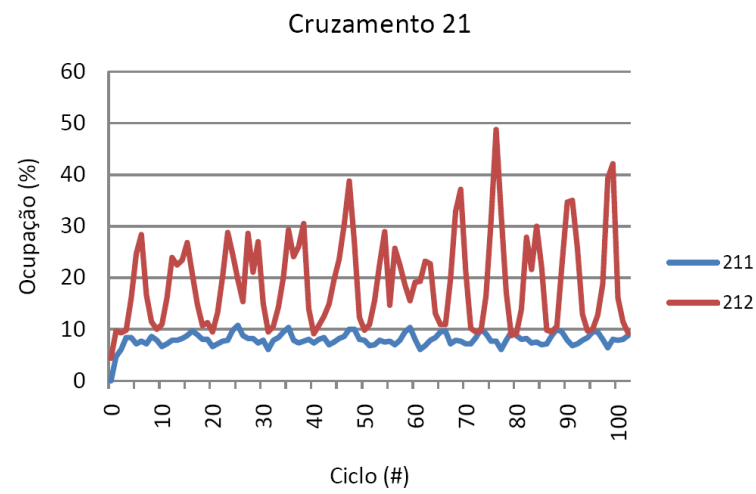

(d) Ocupações do detector em relação ciclo (\%), cruzamento 21, LQR

Figura 2. Desempenho dos controles em tempo fixo e em tempo real LQR para faixa de chegada constante, volume de $750 \mathrm{vph}$. 


$$
\begin{gathered}
g_{211}=g_{211}^{N}+2 \times\left(x_{211}+x_{221}+x_{231}\right) \\
g_{212}=g_{212}^{N}+2 \times x_{212}
\end{gathered}
$$

Ou seja, há três fluxos somando-se para definir o verde do segmento 211, que correspondem às estimativas obtidas a partir das ocupações dos detectores (Figura 3) ao longo da via horizontal inferior da Figura 1. Já para o segmento 212, apenas a contribuição do detector localizado no próprio segmento consegue impactar o controle. Devido ao desbalanceamento, o valor de ocupação no segmento prejudicado (212) tem de crescer muito para conseguir contrabalançar o efeito dos três detectores associados ao segmento 211. Entretanto, quando a fração torna-se suficientemente grande para escoar a fila formada, a ocupação cai novamente e volta a cair a fração de verde de 211 , gerando o comportamento oscilatório.

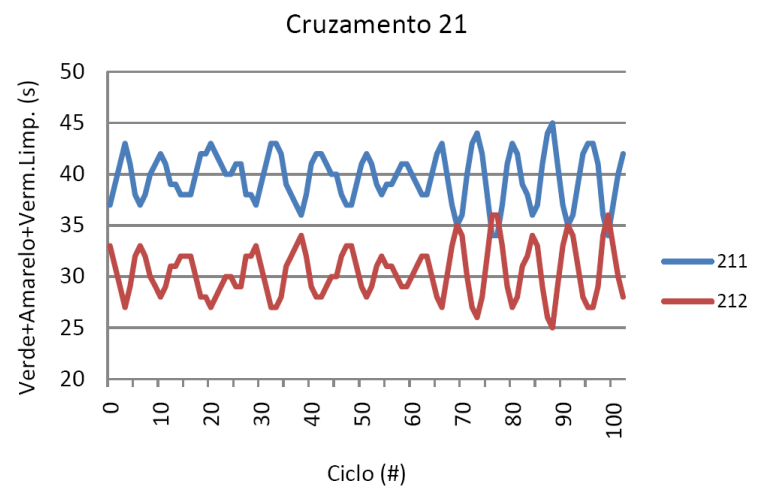

Figura 3. Estágios calculados via LQR com base nas ocupações, cruzamento 21

Conforme destacado anteriormente, a aplicação prática do método LQR requer ajustes de parâmetros adicionais para compensação dos efeitos aqui apresentados. Dois parâmetros são fornecidos para esta finalidade: verdes nominais e fator de importância do segmento.

Para verificar a eficácia do ajuste por tentativa-e-erro desses parâmetros, foram executados vários experimentos de simulação com diversas combinações obtidas por intuição do operador. Ao final, obteve-se desempenho muito similar ao Tempo Fixo com os seguintes parâmetros:

$$
\begin{gathered}
g_{111}^{N}=47.6 \mathrm{~s}, \quad g_{112}^{N}=20.4 \mathrm{~s} \\
g_{121}^{N}=23.8 \mathrm{~s}, \quad g_{122}^{N}=44.2 \mathrm{~s} \\
g_{211}^{N}=20.4 \mathrm{~s}, g_{212}^{N}=47.6 \mathrm{~s} \\
F I_{112}=0.25 \\
F I_{212}=1.6
\end{gathered}
$$

em que,

FI: fatores de importância dos respectivos segmentos. As frações de verde correspondentes são $j_{11}=\{.7, .3\}, j_{12}=$ $\{.35, .65\}, j_{21}=\{.3, .7\}$, com $j_{i k}$ representando as frações dos cruzamentos de acordo com a numeração da Figura 1. Com tais ajustes, o controle tornou-se bem mais estável conforme mostra o gráfico dos tempos de estágio e entreverdes na Figura 4.

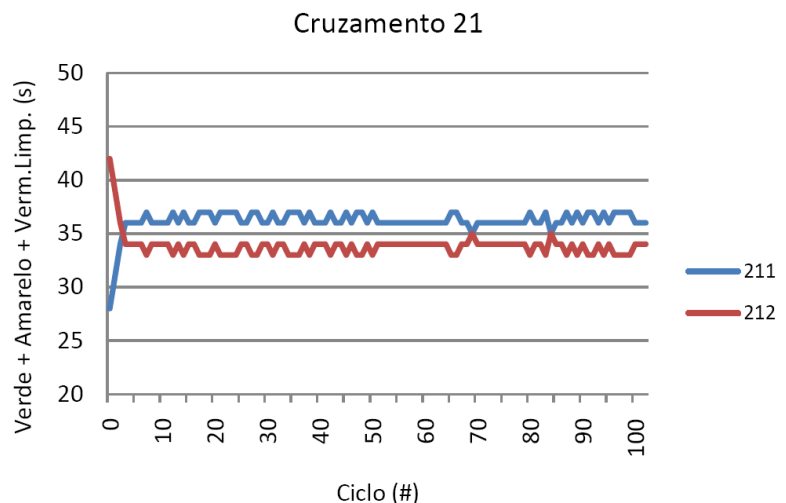

Figura 4. Estágios calculados via LQR com ajustes nos fatores de importância e verde nominais

Também, os indicadores de desempenho mostrados na Tabela 1 confirmam o melhor desempenho do LQR após os ajustes realizados.

Apesar de tratar-se de um caso específico e bastante idealizado, os resultados obtidos confirmam conclusões obtidas em outros estudos a respeito da estratégia TUC. Em resumo:

- O controle LQR puro nem sempre apresenta bom desempenho, necessitando de ajustes complementares;

- Os ajustes são empíricos e por tentativa-e-erro. Não há método sistemático de ajuste devido à forma do problema, no qual o modelo para projeto de controle não corresponde à verdadeira dinâmica do tráfego veicular;

- O resultado do ajuste, conforme visto acima, nem sempre tem uma interpretação física evidente (por que verdes nominais tão díspares? Qual a interrelação entre verdes nominais e fatores de importância?); e

- Porém, uma vez ajustado, o controle LQR por amostragem cíclica desempenha bem a tarefa de controle. No caso acima, praticamente igualou-se ao desempenho do controle ótimo (frações iguais e constantes).

\begin{tabular}{|c|c|c|c|c|c|c|}
\hline & \multicolumn{2}{|l|}{ Atraso } & \multicolumn{2}{|l|}{ Paradas } & \multicolumn{2}{|c|}{ Tempo Total de Viagem } \\
\hline & $(\mathrm{s} / \mathrm{km})$ & $\%$ & $(\# / v e h / k m)$ & $\%$ & (h) & $\%$ \\
\hline Tempo Fixo & 90 & - & 17 & - & 133 & - \\
\hline LQR & 118 & +30 & 21 & +24 & 154 & +16 \\
\hline LQRajs & 91 & +1 & 17 & 0 & 133 & 0 \\
\hline
\end{tabular}

Tais observações motivam a formulação de uma metodologia de ajuste do procedimento LQR que seja embasada em interpretações físicas dos fenômenos do tráfego e que possa ser reproduzida facilmente em qualquer situação, levando a bom desempenho do controle por amostragem cí-

Tabela 1. Desempenho do controle, taxas de chegadas constantes, volume de $750 \mathrm{vph}$ 


$$
\left[\begin{array}{l}
g_{111} \\
g_{112} \\
g_{121} \\
g_{122} \\
g_{131} \\
g_{132} \\
g_{211} \\
g_{212} \\
g_{221} \\
g_{222} \\
g_{231} \\
g_{232}
\end{array}\right]=g^{N}-\left[\begin{array}{cccccccccccc}
-2 & 0 & 0 & 0 & 0 & 0 & 0 & 0 & 0 & 0 & 0 & 0 \\
0 & -2 & 0 & 0 & 0 & 0 & 0 & 0 & 0 & 0 & 0 & 0 \\
0 & 0 & -2 & 0 & 0 & 0 & 0 & 0 & 0 & 0 & 0 & 0 \\
0 & 0 & 0 & -2 & 0 & 0 & 0 & 0 & 0 & 0 & 0 & 0 \\
0 & 0 & -2 & 0 & -2 & 0 & 0 & 0 & 0 & 0 & 0 & 0 \\
0 & 0 & 0 & 0 & 0 & -2 & 0 & 0 & 0 & 0 & 0 & -2 \\
0 & 0 & 0 & 0 & 0 & 0 & -2 & 0 & 0 & 0 & 0 & 0 \\
0 & 0 & 0 & 0 & 0 & 0 & 0 & -2 & 0 & 0 & 0 & 0 \\
0 & 0 & 0 & 0 & 0 & 0 & 0 & 0 & -2 & 0 & -2 & 0 \\
0 & 0 & 0 & -2 & 0 & 0 & 0 & 0 & 0 & -2 & 0 & 0 \\
0 & 0 & 0 & 0 & 0 & 0 & 0 & 0 & 0 & 0 & -2 & 0 \\
0 & 0 & 0 & 0 & 0 & 0 & 0 & 0 & 0 & 0 & 0 & -2
\end{array}\right]
$$

clica em tempo real. A próxima seção apresenta o método proposto neste trabalho para tal finalidade.

\section{PROPOSTA DE NOVO MÉTODO DE CÁLCULO}

Duas observações servem de base para o método proposto. Primeiro, a discussão em torno das Eqs. (4)-(5) indicou o aspecto estrutural do desbalanceamento de $L$, ou seja, das contribuições dos fluxos no cálculo de certos cruzamentos. Segundo, verifica-se na prática que as medidas de ocupações tendem a ser mais afetadas por pequenas flutuações do tráfego do que medidas de fluxos (ou contagens). As consequências de tais observações na formulação do controle são discutidas a seguir.

\subsection{Balanceamento da matriz $L$}

A forma geral da lei de controle (3) apresenta, conforme discutido acima, certos desbalanceamentos da matriz $L$, isto é, o número de detectores que contribuem para o cálculo das frações de verde de um cruzamento pode, em geral, ser desigual. Tal propriedade da forma de $L$ está de acordo com a finalidade original do método TUC, qual seja, o controle de malhas sobre-saturadas. Em tais malhas, essa propriedade auxilia no efeito de gating, ou represamento, dificultando o ingresso de mais veículos em uma malha já congestionada. Em consequência, ocorre uma proteção natural da capacidade interna das vias e evita-se o bloqueio geral da malha interna. Porém, em situações subsaturadas, o desbalanceamento de $L$ provoca as distorções discutidas acima.

Para corrigir esse problema, propõe-se o cancelamento de certos termos de $L$ para igualar as contribuições de cada aproximação. $\mathrm{O}$ procedimento é baseado na configuração da malha viária, e consiste em fazer com que cada cruzamento tenha um mesmo número de detectores sendo considerados para o cálculo das frações de verde. Assim, por exemplo, com referência ao controle da malha viária I, definem-se para os cruzamentos 11,12 e 13 as seguintes contribuições para o cálculo:

$$
\begin{array}{cc}
g_{111}=f\left(x_{111}\right) ; & g_{112}=f\left(x_{112}\right) \\
g_{121}=f\left(x_{112}\right) ; & g_{122}=f\left(x_{122}\right) \\
g_{113}=f\left(x_{112}, x_{113}\right) ; & g_{132}=f\left(x_{132}, x_{232}\right)
\end{array}
$$

e assim por diante para todos os cruzamentos da malha viária.
Aplicando o balanceamento a todos os cruzamentos, o controle (3) passa a ter a forma apresentada na Eq. (6). Antes de avaliar o efeito do balanceamento no desempenho do controle, apresenta-se a seguir o segundo elemento da proposta.

\subsection{Uso de contagens e ocupações}

Outra modificação importante para cálculo do controle em relação ao LQR original refere-se ao tratamento das medições feitas por detectores. Uma vez que as ocupações flutuam mais do que contagens quando os volumes de tráfego são baixos, oscilações indesejáveis podem aparecer quando somente ocupações são medidas. Tal efeito não é importante em situações congestionadas, pois os detectores têm valores consistentemente altos nesse caso. A ideia, então, é usar uma combinação linear de contagens de veículos efetivamente realizadas e das estimativas obtidas a partir das medidas de ocupação com o uso das funções não-lineares $f_{z}(\cdot)$ do método original.

Com base na discussão apresentada, adota-se a seguinte forma para a estimação do número de veículos em um segmento ao longo de um ciclo:

$$
\hat{x}_{i}(k)=o_{i}(k) f_{z}\left(o_{i}(k)\right)+\left(1-o_{i}(k)\right) n_{i}(k),
$$

em que,
$\hat{x}_{i}(k)$ : fluxo estimado no segmento $i$
$o_{i}(\cdot)$ : ocupação temporal medida em campo; e
$n_{i}(\cdot)$ : fluxo medido em um ciclo.

Observa-se que quando a ocupação é baixa a primeira parcela da Eq. (7) é dominada pela segunda parcela, e viceversa. Pode-se dizer que há confiança na contagem de veículos $n_{i}$ como medida do fluxo em um ciclo quando a ocupação é baixa. Por outro lado, se a ocupação é alta, a contagem de veículos já não representa necessariamente o número de veículos no segmento, pois o número de veículos efetivamente contados corresponde aos que saíram do segmento durante o verde, abrindo espaço para novos veículos avançarem sobre o detector e serem contabilizados. Não é possível, nesse caso, contar veículos que estão a montante do detector.

Como é usual em sistemas de controle, faz-se uma filtragem das medidas dos detectores. Embora não mencionado anteriormente, as ocupações no método TUC são a média das duas últimas medidas. Com relação às contagens, adota-se $n_{i}(k)$ como a média das três últimas amostras dos de- 
tectores. Os resultados comparativos do método proposto com respeito aos demais métodos abordados anteriormente são examinados a seguir.

\section{RESULTADOS DE SIMULAÇÃO}

Para avaliação da proposta de controle, foram realizadas simulações com duas malhas viárias, uma das quais já apresentada na Figura 1. A outra malha viária, apresentada na Figura 5, é mais propícia a congestionamentos internos e, portanto, menos vantajosa para o controle em tempo fixo. Nessa malha as taxas de conversão foram definidas como sendo de $10 \%$ a $20 \%$ para giros à esquerda e à direita, ao contrário do cenário sem giros da primeira malha.

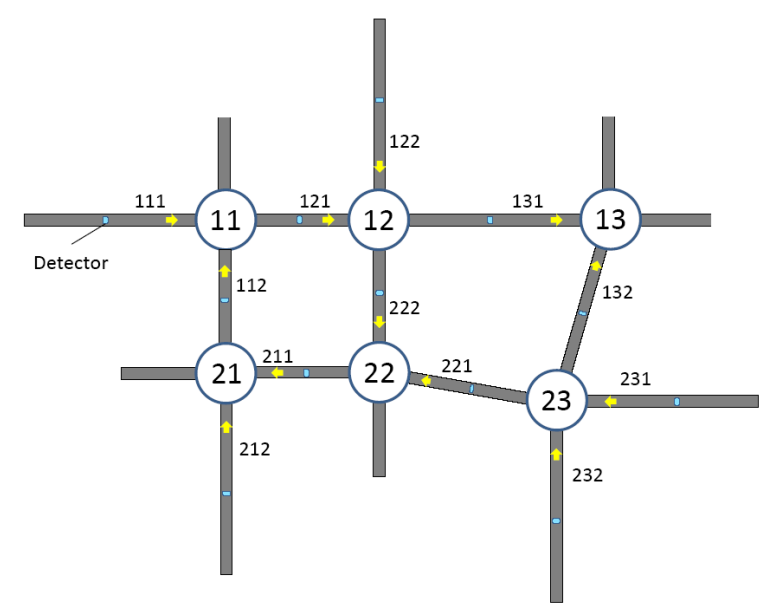

Figura 5. Malha viária II, com vias curtas e desiguais, propícias a congestionamentos internos

\subsection{Chegadas constantes}

As simulações com chegadas constantes, assim como na Seção 3.2, servem para verificar o desempenho do controle em tempo real quando não há variação nos headways de chegadas. Duas condições foram simuladas:

- Fluxos de $750 \mathrm{vph}$ em todas as entradas para malha viária I e de 700 vph para a malha viária II;

- Fluxos alternados da seguinte forma:

- Durante $20 \mathrm{~min}$, vigoram os fluxos de $750 \mathrm{vph} \mathrm{e}$ $700 \mathrm{vph}$ para as malhas I e II, respectivamente; e

- Nos próximos $20 \mathrm{~min}$, vigoram na malha I fluxos de 938 vph nos segmentos 1111 e 1231 e de 625 vph dos demais segmentos de entrada, e de 810 vph e 540 vph na malha II;

- Os valores foram escolhidos para alternar uma situação com demanda menor que a capacidade (e fluxos iguais em todas as entradas) com outra na qual os fluxos são desbalanceados e há sobresaturação nos segmentos $l_{111}$ e $l_{231}$, gerando congestionamentos temporários nas respectivas malhas viárias.

O regime de fluxos alternados serve para avaliação de possíveis ganhos do controle em tempo real em relação ao tempo fixo quando mudam expressivamente as médias de fluxo. Observe-se que, para os fluxos de $\{938,625\} \mathrm{vph}$ na malha I, as frações de verde para o tempo fixo deveriam ser de $60 \%$ para os segmentos horizontais e $40 \%$ para os verticais. Para a malha II, as frações ótimas são diversas, em função das taxas de conversão diferentes de $\{0,1\}$ no interior da malha. Ao manter as frações inalteradas, simula-se a condição de desajuste do tempo fixo.

$\mathrm{Na}$ Tabela 2 são apresentados os indicadores de desempenho para as chegadas constantes, alguns dos quais já mostrados anteriormente. Somente $\mathrm{LQR}_{a j s}$ não é simulado na malha II por não ter sido realizada a sintonia de parâmetros neste caso. Como se observa, o desempenho do controle proposto $\mathrm{LQR}_{b a l}$ é igual ao do tempo fixo para a malha I e superior para a malha II. Ainda que o desempenho do LQR ajustado também seja muito similar para a malha I, permanece o problema da sintonia dos valores desse método. Menciona-se também o melhor desempenho relativo, para a malha II, do método LQR puro. Tal se deve à sintonia menos adequada do tempo fixo devido às conversões internas e ao congestionamento temporário de alguns segmentos que favorece a metodologia de cálculo LQR na medida em que as condições aproximam-se mais das hipóteses do modelo store-and-forward.

A Tabela 3 apresenta os resultados para os volumes alternados com taxas constantes. Como se esperava, neste caso o tempo fixo desempenha pior que o tempo real, pois se encontra mal ajustado. Surpreende o bom desempenho do método LQR puro na malha viária II, corroborando o melhor casamento entre a condição simulada sobre-saturada e os pressupostos do modelo. De todo modo, LQR continua apresentando desempenho muito ruim para a malha viária I, que opera fora da saturação.

\subsection{Distribuição exponencial dos intervalos entre chegadas}

Adotou-se a distribuição exponencial dos intervalos entre chegadas para verificar como o controle em tempo real lida com as variações nos headways entre chegadas. Neste caso, apenas a taxa de chegada com média constante de $750 \mathrm{vph}$ foi simulada. Os resultados estão na Tabela 4. Como pode ser visto, o controle de tempo fixo volta a ter o melhor desempenho para a malha viária I, e na malha II o controle

Tabela 2. Desempenho do controle para malha viária I, taxas de chegadas constantes, volume de 750 vph

\begin{tabular}{|c|c|c|c|c|c|c|c|c|}
\hline & \multicolumn{2}{|l|}{ Atraso } & \multicolumn{2}{|l|}{ Fluxo } & \multicolumn{2}{|l|}{ Paradas } & \multicolumn{2}{|c|}{ Tempo Total de Viagem } \\
\hline & $(\mathrm{s} / \mathrm{km})$ & $\%$ & $(v e h / h)$ & $\%$ & (\#/veh/km) & $\%$ & (h) & $\%$ \\
\hline \multicolumn{9}{|c|}{ Malha Viária I } \\
\hline Tempo Fixo & 90 & - & 3716 & - & 17 & - & 133 & - \\
\hline LQR & 118 & 30 & 3709 & $<1$ & 21 & 24 & 154 & 16 \\
\hline $\mathrm{LQR}_{a j s}$ & 91 & 1 & 3715 & $<1$ & 17 & 0 & 134 & $<1$ \\
\hline $\mathrm{LQR}_{b a l}$ & 90 & 0 & 3716 & 0 & 17 & 0 & 133 & 0 \\
\hline \multicolumn{9}{|c|}{ Malha Viária II } \\
\hline Tempo Fixo & 239 & - & 3430 & - & 18 & - & 194 & - \\
\hline LQR & 250 & +5 & 3442 & $<1$ & 50 & +178 & 197 & +2 \\
\hline $\mathrm{LQR}_{b a l}$ & 219 & -8 & 3429 & $<1$ & 46 & +155 & 182 & -6 \\
\hline
\end{tabular}


Tabela 3. Desempenho para taxas de chegadas constantes, volumes alternados a cada 20 min

\begin{tabular}{|c|c|c|c|c|c|c|c|c|}
\hline & \multicolumn{2}{|l|}{ Atraso } & \multicolumn{2}{|l|}{ Fluxo } & \multicolumn{2}{|l|}{ Paradas } & \multicolumn{2}{|c|}{ Tempo Total de Viagem } \\
\hline & $(\mathrm{s} / \mathrm{km})$ & $\%$ & $(v e h / h)$ & $\%$ & (\#/veh/km) & $\%$ & (h) & $\%$ \\
\hline \multicolumn{9}{|c|}{ Malha Viária I } \\
\hline Tempo Fixo & 111 & - & 3714 & - & 20 & - & 161 & - \\
\hline LQR & 151 & 36 & 3694 & $<1$ & 25 & 25 & 185 & 15 \\
\hline $\mathrm{LQR}_{a d j}$ & 97 & -13 & 3715 & $<1$ & 18 & -10 & 139 & -14 \\
\hline $\mathrm{LQR}_{b a l}$ & 91 & -18 & 3715 & $<1$ & 17 & -15 & 137 & -15 \\
\hline \multicolumn{9}{|c|}{ Malha Viária II } \\
\hline Tempo Fixo & 213 & - & 3106 & - & 48 & - & 168 & - \\
\hline LQR & 180 & -15 & 3213 & +3 & 37 & -23 & 145 & -14 \\
\hline $\mathrm{LQR}_{b a l}$ & 188 & -11 & 3136 & -1 & 42 & -14 & 151 & -7 \\
\hline
\end{tabular}

Tabela 4. Desempenho do controle para taxas de chegadas exponenciais, volume de 750 vph na malha I e 700 vph na malha II

\begin{tabular}{|c|c|c|c|c|c|c|c|c|}
\hline & \multicolumn{2}{|l|}{ Atraso } & \multicolumn{2}{|l|}{ Fluxo } & \multicolumn{2}{|l|}{ Paradas } & \multicolumn{2}{|c|}{ Tempo Total de Viagem } \\
\hline & $(\mathrm{s} / \mathrm{km})$ & $\%$ & $(\mathrm{veh} / \mathrm{h})$ & $\%$ & (\#/veh/km) & $\%$ & (h) & $\%$ \\
\hline \multicolumn{9}{|c|}{ Malha Viária I } \\
\hline Tempo Fixo & 112 & - & 3703 & - & 20 & - & 151 & - \\
\hline LQR & 160 & +43 & 3675 & $<1$ & 27 & 35 & 188 & 25 \\
\hline $\mathrm{LQR}_{a d j}$ & 128 & +14 & 3716 & $<1$ & 22 & 10 & 166 & 10 \\
\hline $\mathrm{LQR}_{b a l}$ & 115 & +3 & 3691 & $<1$ & 20 & 0 & 154 & 2 \\
\hline \multicolumn{9}{|c|}{ Malha Viária II } \\
\hline Tempo Fixo & 300 & - & 3384 & - & 22 & - & 229 & - \\
\hline LQR & 303 & +1 & 3361 & $<1$ & 63 & +186 & 225 & -2 \\
\hline $\mathrm{LQR}_{b a l}$ & 286 & -5 & 3353 & $<1$ & 61 & +177 & 219 & -4 \\
\hline
\end{tabular}

LQR puro é de novo equiparável ao tempo fixo. Em ambos os casos, $\mathrm{LQR}_{b a l}$ apresenta comportamento similar ao tempo fixo. A disparidade do número de paradas na malha II ocorre devido a certas vias apresentarem, para o tempo fixo, paradas mais longas, porém menos frequentes do que observado sob os controles LQR.

\section{CONCLUSÕES}

O método proposto neste trabalho mostrou-se, para a maioria dos casos estudados, superior ao controle originalmente proposto na estratégia TUC. Considerando-se, ainda, que o procedimento de correção do mecanismo básico de cálculo é sistemático e simples de ser adotado em contraste com sintonia por tentativa-e-erro do método anterior, tem-se vantagens significativas com o novo método.

Apesar dos bons resultados obtidos, é preciso considerar a limitação dos casos considerados. Foram estudadas malhas viárias simples em condições de fluxo onde somente havia demandas oriundas das entradas das vias, sem polos geradores de viagens internos às malhas (grandes estacionamentos, p. ex.). Também, não foi abordada a interação do método com controles de defasagens entre cruzamentos próximos e de ciclo. Assim, embora promissor, o método deverá ser estudado analiticamente e por experimentos de simulação e de campo para comprovação de sua eficácia.

\section{REFERÊNCIAS}

Baras, J.; W. Levine e T. Lin (1979) Discrete time point processes in urban traffic queue estimation. IEEE Transactions on Automatic Control AC-24, p. 12-27.

Diakaki, C. (1999) Integrated control of traffic flow in corridor networks. thesis $(\mathrm{PhD})$, Department of Production Engineering and Management, Technical University of Crete, Chania, Greece.

Diakaki, C.; V. Dinopoulou; K. Aboudolas; M. Papageorgiou; E. BenShabat; E. Seider e A. Leibov (2003) Extensions and new applica- tions of the traffic signal control strategy TUC. Transportation Research Record, v. 1856, p. 202-216.

Diakaki, C. M.; M. Papageorgiou e K. Aboudolas (2002) A multivariable regulator approach to traffic responsive network-wide signal control. Control Engineering Practice, v. 10, p. 183-195.

Dorato, P.; C. Abdallah e V. Cerone (1995) Linear- quadratic control: An introduction. Prentice Hall, New Jersey, USA.

Gallego, J.-L.; J. L. Farges e J. J. Henry (1997) Traffic queue estimation. Soc. de Estadística e Investigación Operativa, v. 5, p. 81-93.

Gartner, N. H. (1985), Demand-responsive traffic signal control research. Transportation Research Part A, v. 19A(5/6), p. 369-373.

Gipps, P. (1981) A behavioural car-following model for computer simulation. Transportation Research Part B: Methodological, v. 15, n. 2, p. 105-111.

Gordon, R. L. e W. Tighe (2005) Traffic control systems handbook, Federal Highway Administration, Washington, DC, EUA.

Kosmatopoulos, E.; M. Papageorgiou; C. Bielefeldt; V. Dinopoulou; R. Morris; J. Mueck; A. Richards e F. Weichenmeier (2006) International comparative field evaluation of a traffic-responsive signal control strategy in three cities. Transportation Research Part A, v. 40, n. 5 , p. 399-413.

Kraus, W.; F. de Souza; R. Carlson; M. Papageorgiou; L. Dantas; E. Camponogara; E. Kosmatopoulos e K. Aboudolas (2010) Cost effective Real-Time traffic signal control using the TUC strategy. Intelligent Transportation Systems Magazine, IEEE, v. 2, n. 4, p. 617.

Mirchandani, P. e L. Head (2001) A real-time traffic signal control system: architecture, algorithms, and analysis. Transportation Research Part C: Emerging Technologies v. 9, n. 6, 415-432.

NCHRP (2010) Adaptive traffic control systems: Domestic and foreign state of practice. National Cooperative Highway Research Program (NCHRP) Synthesis 403, Transportation Research Board, Washington, DC, USA.

Robertson, D. I. e R. D. Bretherton (1991) Optimizing networks of traffic signals in real time - The SCOOT Method. IEEE Transactions on Vehicular Technology.

Souza, F. A.; V. Peccin e E. Camponogara (2010) Distributed model predictive control applied to urban traffic networks. IEEE $6^{\text {th }}$ Annual Conference on Automation Science and Engineering, Toronto, Canada.

TSS (2009) Aimsun ng - the integrated transport modeling software, TSS Website. Disponível em: <http://www.aimsun.com>.

Webster, F. V. (1969) Traffic signal settings, road research technical paper no. 39, Department of Scientific and Industrial Research. Road Research Laboratory, London, UK. 